\title{
GEOMETRY OF ISOPARAMETRIC NULL HYPERSURFACES OF LORENTZIAN MANIFOLDS
}

\author{
SAMUEL SSEKAJJA ${ }^{1}$
}

\begin{abstract}
We define two types of null hypersurfaces as; isoparametric and quasi isoparametric null hypersurfaces of Lorentzian space forms, based on the two shape operators associated with a null hypersurface. We prove that; on any screen conformal isoparametric null hypersurface, the screen geodesics lie on circles in the ambient space. Furthermore, we prove that the screen distributions of isoparametric (or quasi-parametric) null hypersurfaces with at most two principal curvatures are generally Riemannian products. Several examples are also given to illustrate the main concepts.
\end{abstract}

\section{INTRODUCTION}

The theory of null submanifolds of a semi-Riemannian manifold is one of the most important topics of differential geometry. More precisely, null hypersurfaces appears in general relativity as models of different types of black hole horizons [13, $14,20]$. The study of non-degenerate submanifolds of semi-Riemannian manifolds has many similarities with the Riemannian submanifolds. However, in case the induced metric on the submanifold is degenerate, the study becomes more difficult and is strikingly different from the study of nondegenerate submanifolds [13, 14]. Some of the pioneering work on null geometry is due to Duggal-Bejancu [13], Duggal- Sahin [14] and Kupeli [24]. Such work motivated many other researchers to invest in the study of null submanifolds, for example $[1,2,3,10,11,15,19]$ and many more references therein.

In the classical theory of non-degenerate submanifolds, a hypersurface is called isoparametric if all its principal curvatures are constant. The first attempt in studying these hypersurfaces was based on a smooth function which was required to be isoparametrc. In fact, a smooth real-valued function $F$ defined on a Riemannian manifold $\tilde{M}$ is called an isoparametric function if both of its classical Beltrami differential parameters $\Delta_{1} F=|\operatorname{grad} F|^{2}$ and $\Delta_{2} F=\Delta F$ (Laplacian of $F$ ) are

2010 Mathematics Subject Classification. Primary 53C25; Secondary 53C40, 53C50.

Key words and phrases. Null hypersurfaces, Isoparametric hypersurfaces, Geodesics. 
smooth functions of $F$ itself. That is, both of the differential parameters are constant on each level set of $F$ (see [25]). An isoparametric family of $\tilde{M}$ is the collection of level sets of a non-constant isoparametric function $F$ on $\tilde{M}$. Thus, in the case where $\tilde{M}$ is a space form, a necessary and sufficient condition for an oriented hypersurface $M \subset \tilde{M}$ to belong to an isoparametric family is that all of its principal curvatures are constant (see [25], Section 3.1 for more details).

Isoparametric hypersurfaces have received considerable attention from many geometers due to theirbeautiful nature, and their theory has been analyzed from several points of view: geometric, algebraic, analytic, and topological. See some examples in $[5,6,8,18,22,25]$ and many more references therein. With the aid of Cartan ideas, [25, Theorem 3.12] proves that any connected isoparametric hypersurface $M^{n}$ of $\mathbb{R}^{n+1}$ is an open subset of a flat hyperplane, a metric hypersphere, or a spherical cylinder $\mathbb{S}^{m} \times \mathbb{R}^{n-m}$. In the hyperbolic space $\mathbb{H}^{n+1}$, they prove that; a connected isoparametric hypersurface is an open subset of a totally geodesic hyperplane, an equidistant hypersurface, a horosphere, a metric hypersphere, or a tube over a totally geodesic submanifold of codimension greater than one in $\mathbb{H}^{n+1}$. In $\mathbb{S}^{n+1}$, they prove $M^{n}$ is an open subset of a standard product of two spheres $\mathbb{S}^{p}(r) \times \mathbb{S}^{q}(s) \subset \mathbb{S}^{n+1}(1) \subset \mathbb{R}^{p+1} \times \mathbb{R}^{q+1}=\mathbb{R}^{n+2}, r^{2}+s^{2}=1$, where $n=p+q$ and $r, s>0$. M. Kimura and S. Maeda [16] give a characterization of isoparametric hypersurfaces in terms of geodesics.

Nomizu [21] began the study of isoparametric hypersurfaces in semi-Riemannian space forms by proving a generalization of Cartans formula for spacelike hypersurfaces in a Lorentzian space form $\tilde{M}(c)$ of constant sectional curvature $c$. As a consequence of this formula, Nomizu showed that a spacelike isoparametric hypersurface in $\tilde{M}(c)$ can have at most two distinct principal curvatures if $c \geq 0$. Later $\mathrm{Li}$ and Xie [17] proved that this conclusion also holds for spacelike isoparametric hypersurfaces in $\tilde{M}(c)$ for $c<0$. Magid [18] studied isoparametric hypersurfaces in Lorentz space whose shape operator is not diagonalizable, and Hahn [12] did an extensive study of isoparametric hypersurfaces in semi-Riemannian space forms of arbitrary signatures. In the null case, M. Navarro, O. Palmas and D. A. Solis [23] have studied null screen isoparametric null in Lorentzian spaceforms, while in [1] the authors study null hypersurfaces with constant screen principal curvatures.

In the present paper, we introduce isoparametric and quasi isoparametric null hypersurfaces in semi-Riemannian space forms. Accordingly, we characterize the above hypersurfaces. The paper is arranged as follows. In Section 2, we give the basic notions null geometry needed for this paper. Section 3 is devoted to isoperimetric null hypersurfaces. In Section 4, we give a characterization of isoparametric 
null hypersurfaces in terms of spacelike and timelike geodesics. Finally, Section 5 is devoted to quasi isoparametric null hypersurfaces and their charaterizations.

\section{Preliminaries}

Let $(\bar{M}, \bar{g})$ be a $(n+2)$-dimensional Lorentzian manifold with index $q \in$ $\{1, \ldots, n+1\}$ and let $M$ be a hypersurface of $\bar{M}$. Let $g$ be the induced tensor field by $\bar{g}$ on $M$. Then, $M$ is called a null hypersurface of $\bar{M}$ if $g$ is of constant rank $n$ [13]. Consider the vector bundle $T M^{\perp}$ whose fibers are defined as

$$
T_{x} M^{\perp}=\left\{Y_{x} \in T_{x} \bar{M}: \bar{g}_{x}\left(X_{x}, Y_{x}\right)=0, \quad \forall X_{x} \in T_{x} M\right\},
$$

for any $x \in M$. Hence, a hypersurface $M$ of $\bar{M}$ is null if and only if $T M^{\perp}$ is a distribution of rank 1 on $M$. Let $M$ be a null hypersurface, we consider the complementary distribution $S(T M)$ to $T M^{\perp}$ in $T M$, which is called a screen distribution. It is well-known that $S(T M)$ is non-degenerate (see [13]). Thus,

$$
T M=S(T M) \perp T M^{\perp} .
$$

As $S(T M)$ is non-degenerate with respect to $\bar{g}$, we have $T \bar{M}=S(T M) \perp$ $S(T M)^{\perp}$, where $S(T M)^{\perp}$ is the complementary vector bundle to $S(T M)$ in $\left.T \bar{M}\right|_{M}$. Let $(M, g)$ be a null hypersurface of $(\bar{M}, \bar{g})$. Then, there exists a unique vector bundle $\operatorname{tr}(T M)$, called the null transversal bundle [13] of $M$ with respect to $S(T M)$, of rank 1 over $M$ such that for any non-zero section $E$ of $T M^{\perp}$ on a coordinate neighborhood $\mathcal{U} \subset M$, there exists a unique section $N$ of $\operatorname{tr}(T M)$ on $\mathcal{U}$ satisfying $\bar{g}(E, N)=1, \bar{g}(N, N)=\bar{g}(N, Z)=0$, for any section $Z$ of $S(T M)$. Consequently, we have the following decomposition of $T \bar{M}$.

$$
\left.T \bar{M}\right|_{M}=S(T M) \perp\left\{T M^{\perp} \oplus \operatorname{tr}(T M)\right\}=T M \oplus \operatorname{tr}(T M) .
$$

Throughout this paper, $\Gamma(\Xi)$ will denote the $\mathcal{F}(M)$-module of differentiable sections of a vector bundle $\Xi$. Let $\nabla$ and $\nabla^{*}$ denote the induced connections on $M$ and $S(T M)$, respectively, and $P$ be the projection of $T M$ onto $S(T M)$, then the local Gauss-Weingarten equations of $M$ and $S(T M)$ are the following [13]

$$
\begin{aligned}
\bar{\nabla}_{X} Y & =\nabla_{X} Y+h(X, Y)=\nabla_{X} Y+B(X, Y) N \\
\bar{\nabla}_{X} N & =-A_{N} X+\nabla_{X}^{t} N=-A_{N} X+\tau(X) N \\
\nabla_{X} P Y & =\nabla_{X}^{*} P Y+h^{*}(X, P Y)=\nabla_{X}^{*} P Y+C(X, P Y) E, \\
\nabla_{X} E & =-A_{E}^{*} X+\nabla_{X}^{* t} E=-A_{E}^{*} X-\tau(X) E, \quad A_{E}^{*} E=0,
\end{aligned}
$$

for all $X, Y \in \Gamma(T M), E \in \Gamma\left(T M^{\perp}\right)$ and $N \in \Gamma(\operatorname{tr}(T M))$, where $\bar{\nabla}$ is the LeviCivita connection on $\bar{M}$. In the above setting, $B$ is the local second fundamental form of $M$ and $C$ is the local second fundamental form on $S(T M) . A_{N}$ and $A_{E}^{*}$ 
are the shape operators on $T M$ and $S(T M)$ respectively, while $\tau$ is a 1-form on $T M$. The above shape operators are related to their local fundamental forms by

$$
g\left(A_{E}^{*} X, Y\right)=B(X, Y), \quad g\left(A_{N} X, P Y\right)=C(X, P Y)
$$

for any $X, Y \in \Gamma(T M)$. Moreover, $\bar{g}\left(A_{E}^{*} X, N\right)=0$, and $\bar{g}\left(A_{N} X, N\right)=0$, for all $X \in \Gamma(T M)$. From these relations, we notice that $A_{E}^{*}$ and $A_{N}$ are both screenvalued operators. Let $\vartheta=\bar{g}(N, \cdot)$ be a 1-form metrically equivalent to $N$ defined on $\bar{M}$. Take $\eta=i^{*} \vartheta$ to be its restriction on $M$, where $i: M \rightarrow \bar{M}$ is the inclusion map. Then it is easy to show that

$$
\left(\nabla_{X} g\right)(Y, Z)=B(X, Y) \eta(Z)+B(X, Z) \eta(Y),
$$

for all $X, Y, Z \in \Gamma(T M)$. Consequently, $\nabla$ is generally not a metric connection with respect to $g$. However, the induced connection $\nabla^{*}$ on $S(T M)$ is a metric connection.

Denote by $\bar{R}, R$ and $R^{*}$ the curvature tensors of the connection $\bar{\nabla}$ on $\bar{M}$, and the induced linear connections $\nabla$ and $\nabla^{*}$ on $M$ and $S(T M)$, respectively. Using the Gauss-Weingarten formulae, we obtain the following Gauss-Codazzi equations for $M$ and $S(T M)$ (see details in [13, 14]).

$$
\begin{aligned}
\bar{R}(X, Y) Z= & R(X, Y) Z+B(X, Z) A_{N} Y-B(Y, Z) A_{N} X \\
& +\left\{\left(\nabla_{X} B\right)(Y, Z)-\left(\nabla_{Y} B\right)(X, Z)+\tau(X) B(Y, Z)\right. \\
& -\tau(Y) B(X, Z)\} N \\
R(X, Y) P Z= & R^{*}(X, Y) P Z+C(X, P Z) A_{E}^{*} Y-C(Y, P Z) A_{E}^{*} X \\
& +\left\{\left(\nabla_{X} C\right)(Y, P Z)-\left(\nabla_{Y} C\right)(X, P Z)-\tau(X) C(Y, P Z)\right. \\
& +\tau(Y) C(X, P Z)\} E \\
R(X, Y) E= & -\nabla_{X}^{*} A_{E}^{*} Y+\nabla_{Y}^{*} A_{E}^{*} X+A_{E}^{*}[X, Y]-\tau(X) A_{E}^{*} Y \\
& +\tau(Y) A_{E}^{*} X+\left\{C\left(Y, A_{E}^{*} X\right)-C\left(X, A_{E}^{*} Y\right)\right. \\
& -2 d \tau(X, Y)\} E \\
\bar{R}(X, Y) N= & -\nabla_{X} A_{N} Y+\nabla_{Y} A_{N} X+A_{N}[X, Y]+\tau(X) A_{N} Y \\
& -\tau(Y) A_{N} X+\left\{B\left(Y, A_{N} X\right)-B\left(X, A_{N} Y\right)\right. \\
& -2 d \tau(X, Y)\} N
\end{aligned}
$$

where $d \tau(X, Y)=X(\tau(Y))-Y(\tau(X))-\tau([X, Y])$, for all $X, Y, Z \in \Gamma(T M)$, $E \in \Gamma\left(T M^{\perp}\right)$ and $N \in \Gamma(\operatorname{tr}(T M))$. A semi-Riemannian manifold $(\bar{M}, \bar{g})$ of constant sectional curvature $c$ is called a semi-Riemannian space form (see [20, p. 
80]) and denoted by $\bar{M}(c)$. The curvature tensor field $\bar{R}$ of $\bar{M}(c)$ is given by

$$
\bar{R}(\bar{X}, \bar{Y}) \bar{Z}=c\{\bar{g}(\bar{Y}, \bar{Z}) \bar{X}-\bar{g}(\bar{X}, \bar{Z}) \bar{Y}\}, \quad \forall \bar{X}, \bar{Y}, \bar{Z} \in \Gamma(T \bar{M}) .
$$

A null hypersurface $(M, g)$ of a semi-Riemannian manifold $(\bar{M}, \bar{g})$ is called;

(1) totally umbilic [13, p. 106] if there exist a smooth function $\rho$ on a coordinate neighborhood $\mathcal{U} \subset M$ such that $A_{E}^{*} X=\rho P X$, or equivalently, $B(X, P Y)=\rho g(X, Y)$. In case $\rho=0$ on $\mathcal{U}$, we say that $M$ is totally geodesic otherwise it is proper totally umbilic in $\bar{M}$.

(2) screen totally umbilic [13, p. 109] if there exist a smooth function $\varrho$ on a coordinate neighborhood $\mathcal{U} \subset M$ such that $A_{N} X=\varrho P X$, or equivalently, $C(X, P Y)=\varrho g(X, Y)$. In case $\varrho=0$ on $\mathcal{U}$, we say that $M$ is screen totally geodesic otherwise it is proper screen totally umbilic in $\bar{M}$.

(3) screen conformal [14, p. 60] if there exist a non-vanishing smooth function $\psi$ on a coordinate neighborhood $\mathcal{U}$ such that $A_{N} X=\psi A_{E}^{*} X$, or equivalently, $C(X, P Y)=\psi B(X, Y)$,

for any $X, Y \in \Gamma(T M)$. For example, a null cone $\Lambda_{0}^{n+1}=\left\{\left(x_{0}, \ldots, x_{n+1}\right) \in\right.$ $\left.\mathbb{R}^{n+2} \mid x_{0}^{2}=\sum_{a=1}^{n+1} x_{a}^{2}, \quad x_{0} \neq 0\right\}$ of $\mathbb{R}_{0}^{n+2}$ is totally umbilic with $\rho=-1$ and it is also screen totally umbilic with $\varrho=-\frac{1}{2 x_{0}^{2}}$. Moreover, $M$ is screeen conformal with $\psi=\frac{1}{2 x_{0}^{2}}$ (see Propositions 5.4 and 5.5 of [13, p. 111-113] for more details). A null Monge hypersurface of $\mathbb{R}_{q}^{n+2}$ is screen conformal with $\psi=\frac{1}{2}$ (see Proposition 6.3 of [13, p. 121]). More examples can be found in the books $[13,14]$.

\section{ISOPARAMETRIC NULL HYPERSURFACES}

In the theory of non-degenerate submanifolds, each hypersurface has only one type of fundamental form with its respective shape operator. Moreover, the second fundamental form and its corresponding shape operator are related by means of the induced metric tensor. In this case, a hypersurface is called isoparametric if it has constant principal curvatures (see, for instance, [25, Chapter 3]). For the degenerate case, each null hypersurface has two types of fundamental forms $B$ and $C$ with their two respective shape operators $A_{N}$ and $A_{E}^{*}$. We see, from (2.7), that there are interrelations between the null and screen second fundamental forms and their respective shape operators. Due to this interrelatedness, we will define isoparametric null hypersurfaces $(M, g)$ based on the pricincipal curvatures from the screen shape operator $A_{E}^{*}$. First, note that the second fundamental form $B$ of a null hypersuface $(M, g)$ is degenerate in addition to the degeneracy of the induced metric tensor $g$. In fact, $B(E, \cdot)=0$ and hence, $A_{E}^{*} E=0$. Consequently, the only principal curvature of a null hypersurface along its normal bundle $T M^{\perp}(\subset$ 
$T M)$ is constant, and equal to zero. Thus, to investigate constancy of principal curvatures (or isoparametricity) on a null hypersurface reduces to investigating the constancy of remaining principal curvatures, with respect to $A_{E}^{*}$, along the screen distribution $S(T M)$. Based on the above, we define the concept of isoparametric null hypersurfaces as follows;

Definition 3.1. A null hypersurface $(M, g)$ immersed in a semi-Riemannian manifold $(\bar{M}, \bar{g})$ is said to be isoparametric if the screen principal curvatures, with respect to $A_{E}^{*}$, are constant along $S(T M)$.

Throughout this paper, we denote by $d$ the number of distinct principal curvatures of $S(T M)$ with respect to $A_{E}^{*}$. For instance, all totally geodesic $n$-dimensional null hypersurfaces are isoparametric, in which $A_{E}^{*}$ has one principal curvature zero with multiplicity $n$. Thus, $d=1$. More explicitly, we have the following example.

Example 3.2. Let $S_{1}^{4}$ be the unit pseudo-sphere of a Minkowski spacetime $R_{1}^{5}$ given by $-t^{2}+x^{2}+y^{2}+z^{2}+w^{2}=1$. Cut $S_{1}^{4}$ by the hypersurface $t-x=0$ and obtain a null surface $M$ of $S_{1}^{4}$ with $\operatorname{Rad} T M$ spanned by a null vector $E=$ $\partial_{t}+\partial_{x}$. Take a screen distribution $S(T M)$ spanned by the unit spacelike vector fields $W_{1}=\left(z^{2}+w^{2}\right)^{-\frac{1}{2}}\left(w \partial_{z}-z \partial_{w}\right)$ and $W_{2}=\left(z^{2}+w^{2}\right)^{-\frac{1}{2}}\left(z \partial_{z}+w \partial_{w}\right)$. A routine computation leads us to $\nabla_{W_{1}} E=0$ and $\nabla_{W_{2}} E=0$. Clearly, $M$ is totally geodesic and hence, isoparametric.

Other none trivial examples of isoparametric null hypersurfaces include the following.

Example 3.3 (Null cone of $\mathbb{R}_{1}^{n+2}$ ). Let $\mathbb{R}_{1}^{n+2}$ be the space $\mathbb{R}^{n+2}$ endowed with a semi-Euclidean metric $\bar{g}(x, y)=-x_{0} y_{0}+\sum_{a=0}^{n+1} x_{a} y_{a}, \quad\left(x=\sum_{A=0}^{n+1} x^{A} \partial x_{A}\right)$, where $\partial x_{A}:=\frac{\partial}{\partial x_{A}}$. Then, the null cone $\Lambda_{0}^{n+1}$ is given by the equation $x_{0}^{2}=$ $\sum_{a=1}^{n+1} x_{a}^{2}, x_{0} \neq 0$. It is well-known (for example see the books [13, 14]) that $\Lambda_{0}^{n+1}$ is a null hypersurface of $\mathbb{R}_{1}^{n+2}$, in which the radical distribution is spanned by a global vector field $E=\sum_{A=0}^{n+1} x_{A} \partial x_{A}$ on $\Lambda_{0}^{n+1}$. The transversal bundle is spanned by a global section $N$ given by $N=\frac{1}{2 x_{0}^{2}}\left\{-x_{0} \partial x_{0}+\sum_{a=1}^{n+1} x_{a} \partial x_{a}\right\}$. Moreover, $E$ being the position vector field, one gets $\bar{\nabla}_{X} E=\nabla_{X} E=X$, for any $X \in \Gamma\left(T \Lambda_{0}^{n+1}\right)$. Consequently, $A_{E}^{*} X+\tau(X) E+X=0$. Noticing that the operator $A_{E}^{*}$ is screen-valved, we infer from the last relation that

$$
A_{E}^{*} X=-P X, \quad \tau(X)=-\bar{g}(X, N)=-\lambda(X),
$$

for any $X \in \Gamma\left(T \Lambda_{0}^{n+1}\right)$. Next, any $X \in \Gamma\left(S\left(T \Lambda_{0}^{n+1}\right)\right)$ is expressed as $X=$ $\sum_{a=1}^{n+1} \tilde{X}_{a} \partial x_{a}$, where $\left\{\tilde{X}_{1}, \ldots, \tilde{X}_{n+1}\right\}$ satisfy $\sum_{a=1}^{n+1} x_{a} \tilde{X}_{a}=0$. From the first relation of (3.1) we can clearly see that $\Lambda_{0}^{n+1}$ is isoparametric with $d=1$. 
Example 3.4. Consider a 6-dimensional space $\bar{M}=\mathbb{R}^{6}$ equiped with a Lorentzian metric $\bar{g}=-\left(\mathrm{d} x^{0}\right)^{2}+\left(\mathrm{d} x^{1}\right)^{2}+e^{2 x^{0}}\left\{\left(\mathrm{~d} x^{2}\right)^{2}+\left(\mathrm{d} x^{3}\right)^{2}\right\}+e^{2 x^{1}}\left\{\left(\mathrm{~d} x^{4}\right)^{2}+\left(\mathrm{d} x^{5}\right)^{2}\right\}$, where $\left(x^{0}, \cdots, x^{5}\right)$ are the usual rectangular coordinates on $\bar{M}$. The non-zero Christoffel coefficients of the Levi-Civita connection of $\bar{g}$ are $\Gamma_{02}^{2}=\Gamma_{03}^{3}=\Gamma_{14}^{4}=$ $\Gamma_{15}^{5}=1, \Gamma_{22}^{0}=\Gamma_{33}^{0}=-e^{2 x^{0}}$ and $\Gamma_{44}^{1}=\Gamma_{55}^{1}=e^{2 x^{1}}$. Consider a hypersurface $M$ of $\bar{M}$ given by $M=\left\{\left(x^{0}, \cdots, x^{5}\right) \in \mathbb{R}^{6}: x^{0}+x^{1}=0\right\}$. Then, $M$ is a null hypersurface with $N=-\frac{1}{2}\left(\partial x^{0}+\partial x^{1}\right)$ and $E=\partial x^{0}-\partial x^{1}$. Also, $S(T M)=$ $\operatorname{Span}\left\{e_{1}, e_{2}, e_{3}, e_{4}\right\}$, where $e_{1}=e^{-2 x^{0}} \partial x^{2}, e_{2}=e^{-2 x^{0}} \partial x^{3}, e_{3}=e^{-2 x^{1}} \partial x^{4}$ and $e_{4}=e^{-2 x^{1}} \partial x^{5}$. Notice that $\left[e_{i}, e_{j}\right]=0$, for all $i, j \in\{1,2,3,4\}$. Hence, $S(T M)$ is integrable. By a straightforward calculation, we have $\nabla_{e_{1}} E=e_{1}, \nabla_{e_{2}} E=e_{2}$, $\nabla_{e_{3}} E=-e_{3}$ and $\nabla_{e_{4}} E=-e_{4}$. It then follows that $A_{E}^{*} e_{1}=-e_{1}, A_{E}^{*} e_{2}=-e_{2}$, $A_{E}^{*} e_{3}=e_{3}$ and $A_{E}^{*} e_{4}=e_{4}$. Thus, -1 and 1 are the two distinct constant principal curvatures of $A_{E}^{*}$ with respect to $S(T M)$, with multiplicity 2 each. Clearly, $M$ is isoparametric with $d=2$.

Next, we characterize isoparametric null hypesurfaces with $d=1$.

Theorem 3.5. Any totally umbilic null hypersurface $(M, g)$ of a semi-Riemannian manifold $(\bar{M}(c), \bar{g})$ such that the one form $\tau$ vanishes on $S(T M)$ is isoparametric.

Proof. As $M$ is totally umbilic in $\bar{M}(c)$, we have $A_{E}^{*} X=\rho P X$, for all $X \in$ $\Gamma(T M)$. Clearly, if $X \in \Gamma(S(T M))$ is a principal vector field of $A_{E}^{*}$ then $\rho$ is the corresponding principal curvature function. It is well-known [13, p. 108] that the function $\rho$ satisfies $E(\rho)+\rho \tau(E)-\rho^{2}=0$ and $P X(\rho)+\rho \tau(P X)=0$, for any $X \in \Gamma(T M)$ and $E \in \Gamma\left(T M^{\perp}\right)$. The result follows from the second differential equation by considering the fact that $\tau$ vanishes on $S(T M)$.

Corollary 3.6. Any totally umbilic isoparametric null hypesurface $(M, g)$ of a semi-Riemannian manifold $(\bar{M}(c), \bar{g})$ is either an open subset of a hyperplane in $\bar{M}$ or $\tau$ vanishes on $S(T M)$.

Next, let $\lambda$ be a smooth principal curvature, with $d>1$, and let $P_{\lambda}$ denote the corresponding principal distribution. Then, we have the following result.

Proposition 3.7. Let $(M, g)$ be a null hypersurface of an indefinite space form $(\bar{M}(c), \bar{g})$. Suppose that $\lambda$ is a smooth principal curvature function of $A_{E}^{*}$ of constant multiplicity $m>1$ on $S(T M)$. Then the principal distribution $P_{\lambda}$ is integrable. Moreover, $\lambda$ is constant along each $P_{\lambda}$ if and only if the 1-form $\tau$ vanishes on $P_{\lambda}$. Furthermore, if $M$ is screen conformal, such that $\tau$ vanishes on $S(T M)$, and the number of distinct principal curvatures of $S(T M)$ is $\leq 2$, then the screen conformality factor $\psi$ is constant along each principal distribution $P_{\lambda}$. 
Proof. In view of relations (2.11) and (2.13), we derive

$$
-\nabla_{X}^{*} A_{E}^{*} Y+\nabla_{Y}^{*} A_{E}^{*} X+A_{E}^{*}[X, Y]-\tau(X) A_{E}^{*} Y+\tau(Y) A_{E}^{*} X=0,
$$

for any $X, Y \in \Gamma(S(T M))$. Next, let us consider $X$ and $Y$ to be linearly independent local vector fields in the principal distribution $P_{\lambda}$, then (3.2) gives

$$
A_{E}^{*}[X, Y]-\lambda[X, Y]=\{X(\lambda)+\lambda \tau(X)\} Y-\{Y(\lambda)+\lambda \tau(Y)\} X .
$$

As the right hand side of (3.3) belongs to $P_{\lambda}$, while the left side is in $P_{\lambda}^{\perp}$, both sides are equal to zero. Thus, $P_{\lambda}$ is integrable by the Frobenius theorem, since $[X, Y]$ belongs to $P_{\lambda}$. Also, as $X$ and $Y$ are linearly independent, we deduce that $X(\lambda)+\lambda \tau(X)$ and $Y(\lambda)+\lambda \tau(Y)$ are both zero on $P_{\lambda}$. Each of these relations implies that $X(\lambda)=0$ if and only if $\tau(X)=0$, for all $X \in \Gamma\left(P_{\lambda}\right)$. Next, we prove the constancy of $\psi$ along each principal distribution when $M$ is screen conformal. First, suppose that the number of distinct screen principal curvatures $d=1$. In this case, $M$ is totally umbilic in $\bar{M}$ with $\rho=\lambda$, where $\lambda$ is the only principal curvature of $S(T M)$ with respect to $A_{E}^{*}$. As $M$ is also screen conformal, we have that $S(T M)$ is totally umbilic with $\varrho=\psi \lambda$. Using (2.12) and the fact that $\bar{M}$ is a space of constant curvature $c$, we have

$$
\begin{aligned}
& \{X(\psi \lambda)-\psi \lambda \tau(X)-c \eta(X)\} g(P Y, P Z) \\
& -\{Y(\psi \lambda)-\psi \lambda \tau(Y)-c \eta(Y)\} g(P X, P Z) \\
& -\psi \lambda\{B(P Y, P Z) \eta(X)-B(P X, P Z) \eta(Y)\}=0,
\end{aligned}
$$

for all $X, Y, Z \in \Gamma(T M)$. Setting $X=P X, Y=P Y$ and $Z=P Y$ in (3.4), we deduce that $P X(\psi \lambda)-\psi \lambda \tau(P X)=0$. As $\tau=0$ on $S(T M)$, we have $\lambda$ is a constant, and thus either $\lambda=0$ or $P X(\psi)=0$. In the first case, that is; $\lambda=0$, we have that both $S(T M)$ and $M$ are totally geodesic. Hence, $\psi$ is an arbitrary constant function. Otherwise, the second case applies which shows that $\psi$ is constant along $S(T M)$. We now turn to the case $d=2$. From relations (2.12) and (2.11), with $\tau=0$ on $S(T M)$, we have

$$
X(\psi) B(Y, P Z)=Y(\psi) B(X, P Z),
$$

for any $X, Y \in \Gamma(S(T M))$ and $Z \in \Gamma(T M)$. Let us denote by $\lambda$ and $\mu$ the two distinct screen principal curvatures. Also, let $P_{\lambda}$ and $P_{\mu}$ be their corresponding principal distributions. Then Lemma 2.5 .6 of [14, p. 79] suggests that $P_{\lambda} \perp P_{\mu}$. Setting $X \in \Gamma\left(P_{\lambda}\right)$ and $Y, P Z \in \Gamma\left(P_{\mu}\right)$ in (3.5), we get

$$
X(\psi) B(Y, P Z)=Y(\psi) B(X, P Z)=\lambda Y(\psi) g(X, P Z)=0 .
$$


Consequently, $\mu X(\psi)=0$. On the other hand, considering (3.4) with $X, Y, Z \in$ $\Gamma\left(P_{\lambda}\right)$, we have $\lambda X(\psi)=0$. If we consider $X(\psi) \neq 0$ in these equations, we get $\mu=\lambda=0$, which makes $M$ totally geodesic. This contradicts the fact that $d=2$. Thus $X(\psi)=0$ for any $X \in \Gamma\left(P_{\lambda}\right)$, which completes the proof.

Remark 3.8. Unlike the non-degenerate case in which if $m>1$ then $\lambda$ is a constant along $P_{\lambda}$ (see Chen [7, p. 102]), the null case is different as the constancy depends on the 1-form $\tau$.

In the case $\lambda$ has constant multiplicity $m>1$ on $S(T M)$, the distribution $P_{\lambda}$ is a foliation on $S(T M)$, which we call the principal foliation corresponding to $\lambda$. Next, we prove that the leaves of a principal foliation are $m$-dimensional totally umbilic submanifolds of $\bar{M}$, and totally geodesic in $S(T M)$. To that end, a leaf $M_{\lambda}$ of $P_{\lambda}$ is said to be totally umbilic in $\bar{M}$ if for each $x \in M_{\lambda}$, there is a realvalued linear function $\phi$ on $T_{x} M_{\lambda}^{\perp}$ such that the shape operator $A_{\nu}$ of $M_{\lambda}$ satisfies $A_{\nu}=\phi I$, for every $\nu$ in $T_{x} M_{\lambda}^{\perp}$. In case $\phi=0$, we say that $M_{\lambda}$ is totally geodesic. With the above in mind, we have the following result.

Theorem 3.9. Let $(M, g)$ be a screen conformal or proper screen totally umbilic null hypersurface of a semi-Riemanian manifold $(\bar{M}(c), \bar{g})$. Suppose that $\lambda$ is a smooth principal curvature function of $A_{E}^{*}$ of constant multiplicity $m>1$ on $S(T M)$. Then, the number $d$ of distinct principal curvatures $\lambda$ is at most 2. The leaves of $P_{\lambda}$ are either $m$-spheres or planes in $\bar{M}(c)$. Moreover, if $\tau$ vanishes on $S(T M)$ and there are two distinct screen principal curvatures, then each leave of $P_{\lambda}$ is a hyperplane in $S(T M)$.

Proof. The first part of this theorem, i.e., the number of distinct principal curvatures $d$ is at most 2, follows easily from the null Cartan-like formula [1, 23]. Next, we will show that a leaf $M_{\lambda}$ is totally umbilic in $\bar{M}$, which shows (see Proposition 36 of O'Neill [20, p. 116]) $M_{\lambda}$ is either an $m$-sphere or plane. First, we note that at each $x \in M_{\lambda}$, the normal subspace $T_{x} M_{\lambda}^{\perp}$ of a leaf $M_{\lambda}$ of the principal foliation $P_{\lambda}$ in $\bar{M}$ is $T_{x} M_{\lambda}^{\perp}=P_{\lambda}^{\perp}(x) \perp\left\{\operatorname{Rad} T_{x} M \oplus \operatorname{tr}\left(T_{x} M\right)\right\}$, where $P_{\lambda}^{\perp}(x)$ is the orthogonal complement of $P_{\lambda}$ in $S\left(T_{x} M\right)$. Let $A_{\nu}$ denote the shape operator of $M_{\lambda}$ in $\bar{M}$, where $\nu$ belongs to $T_{x} M_{\lambda}^{\perp}$. If $\nu$ belongs to the hyperbolic bundle $\operatorname{Rad} T_{x} M \oplus \operatorname{tr}\left(T_{x} M\right)$, then we can write $\nu=a E+b N$, for some smooth non-vanishing functions $a$ and $b$. From this, we deduce that $A_{\nu} X=a A_{E}^{*} X+b A_{N} X=a \lambda X+b A_{N} X$, for any $X$ in $\Gamma\left(P_{\lambda}\right)$. Thus, a leaf $M_{\lambda}$ is totally umbilic if $M$ is screen conformal or screen totally umbilic. Let $v \in \Gamma\left(P_{\lambda}^{\perp}(x)\right)$ be a unit principle vector of $A_{E}^{*}$, with corresponding principle curvature $\mu \neq \lambda$. Let us extend $v$ to a vector field $Y \in \Gamma\left(P_{\lambda}^{\perp}\right)$ on a neighborhood $\mathcal{U}$ 
of $x$. Then, there exists a unique vector field $Z \in \Gamma\left(P_{\lambda}^{\perp}\right)$ such that $g(Y, Z)=0$ (see [25]), and that

$$
A_{E}^{*} Y=\mu Y+Z,
$$

for some smooth function $\mu$ on $\mathcal{U}$. This supported by the fact that $P_{\lambda}^{\perp}$ is invariant under $A_{E}^{*}$, even though the eigenvalues of $A_{E}^{*}$ need not be smooth. Let $X$ be a vector field in $P_{\lambda}$ on the neighborhood $\mathcal{U}$. Since the vector field $Z=0$ at $x$, one can easily show that $\nabla_{X}^{*} Z \in \Gamma\left(P_{\lambda}^{\perp}\right)$ at $x$. Hence, by (2.11) and (3.6), we derive

$$
\begin{gathered}
\{X(\mu)+\mu \tau(X)\} Y-\{Y(\lambda)+\lambda \tau(Y)\} X+\nabla_{X}^{*} Z \\
=\left(A_{E}^{*}-\mu I\right) \nabla_{X}^{*} Y-\left(A_{E}^{*}-\lambda I\right) \nabla_{Y}^{*} X,
\end{gathered}
$$

which gives us

$$
-\frac{Y(\lambda)+\lambda \tau(Y)}{\lambda-\mu} X
$$

as the $P_{\lambda}$-component of $\nabla_{X}^{*} Y$. Furthermore, from relations (2.3) and (2.5), we have $\bar{\nabla}_{X} Y=\nabla_{X}^{*} Y+g\left(A_{N} X, Y\right) E+g\left(A_{E}^{*} X, Y\right) N$. As $M$ is either screen conformal or proper screen totally umbilical in $\bar{M}$, we see, by (3.6), that $g\left(A_{N} X, Y\right)=$

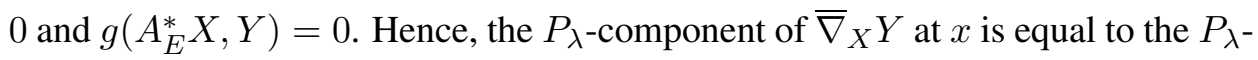
component of $\nabla_{X}^{*} Y$ at $x$, which is given by (3.8). Since $v=Y$ at $x$, the vector field $-A_{v} X$ is by definition equal to the $P_{\lambda}$-component of $\bar{\nabla}_{X} Y$, and so we have

$$
A_{v} X=\frac{Y(\lambda)+\lambda \tau(Y)}{\lambda-\mu} X
$$

That is, each leaf of $P_{\lambda}$ is totally umbilic in $\bar{M}$. Next, we prove the last part of the theorem. As the number of screen principal curvatures is atmost 2, we have, by Lemma 2.5 .6 of [14, p. 79], that $P_{\lambda} \perp P_{\mu}$, where $\lambda$ and $\mu$ are the two distinct principal curvatures of $S(T M)$. Since $\tau=0$ on $S(T M)$, it is easy to show, from (2.11), that $\nabla_{X}^{*} Y \in \Gamma\left(P_{\lambda}\right)$ for any $X, Y \in \Gamma\left(P_{\lambda}\right)$. Consequently, $g\left(\nabla_{X} Y, Z\right)=0$ implying a leaf of $P_{\lambda}$ is totally geodesic in $S(T M)$. The same is true for the leaves of $P_{\mu}$. Therefore, each leaf of $P_{\lambda}$ or $P_{\mu}$ is an hyperplane in $S(T M)$, which completes the proof.

Note that, with an $m$-sphere in a Lorentzian manifold $\mathbb{R}_{1}^{n+2}$, we mean a hypersphere in the Euclidean space $\mathbb{R}^{m+1}$.

As an example, we have the following.

Example 3.10. Let us consider the null cone of Example 3.3, in which both $\Lambda_{0}^{n+1}$ and $S\left(T \Lambda_{0}^{n+1}\right)$ are totally umbilic with $\rho=-1$ and $\varrho=-\frac{1}{2\left(x^{0}\right)^{2}}$, respectively (see $\left[13\right.$, p. 112] for more details). From the Gauss equations of $\Lambda_{0}^{n+1}$ and $S\left(T \Lambda_{0}^{n+1}\right)$, 
we have $\bar{\nabla}_{X} Y=\nabla_{X}^{*} Y-g(X, Y)\left(1 / 2\left(x^{0}\right)^{2} E+N\right)$, for any $X, Y \in \Gamma\left(T M_{\lambda}\right)$. Here, $M_{\lambda}$ is a leaf of $S\left(T \Lambda_{0}^{n+1}\right)$ and $\nabla^{*}$ is the Levi-Civita connection on $M_{\lambda}$. Thus, $M_{\lambda}$ is totally umbilic in $\mathbb{R}_{1}^{n+2}$. As $x^{0} \neq 0$, we may assume that $x^{0}>0$ (for $x^{0}<0$, we proceed in the same way) and consider in the normal bundle $T M_{\lambda}^{\perp}$, the vector fields $v_{1}=1 / 2 x^{0} E+x^{0} N$ and $v_{2}=-1 / 2 x^{0} E+x^{0} N$. Observe that $\left\{v_{1}, v_{2}\right\}$ is an orthonormal basis, with $v_{1}$ and $v_{2}$ spacelike and timelike vector fields, respectively. By a simple calculation, using the expressions of $E, N$ and the second relation in (3.1), we get $\bar{\nabla}_{X} v_{2}=1 / 2 x^{0} A_{E}^{*} X-x^{0} A_{N} X=0$. Hence, $M_{\lambda}$ is embedded in $\mathbb{R}^{n+1}$. As $M_{\lambda}$ is totally umbilical but not totally geodesic immersed in the Euclidean space $\mathbb{R}^{n+1}$, by Proposition 5.6 of [13, p. 113] (or Proposition 36 of $\left[20\right.$, p. 116] ), we see that $M_{\lambda}$ lies on hypersphere in $\mathbb{R}^{n+1}$. Hence, $M_{\lambda}$ is an $n$-sphere in $\mathbb{R}_{1}^{n+2}$.

Let $M$ be a null hypersurface of a semi-Riemannian manifold $\bar{M}$. The mean curvature of $M$ is denoted by $\mathcal{H}$, and given by $\mathcal{H}=\frac{1}{n} \operatorname{tr}_{\mid S(T M)} B$. Accordingly, $M$ is said to be of constant mean curvature if $\mathcal{H}$ is a constant function. In the special case: $\mathcal{H}=0$, we say that $M$ is minimal null hypersurface. For instance, all totally geodesic null hypersurfaces are trivially minimal.

Theorem 3.11. Let $(M, g)$ be a screen conformal null hypersurface of $(\bar{M}(c), \bar{g})$ with compact leaves $M^{*}$. Suppose that $M$ has constant mean curvature and two principal curvatures $\lambda$ and $\mu$ along its screen distribution, with multiplicities $m$ and $n-m$, respectively. If the 1 -form $\tau$ vanishes on $S(T M)$ and $1<m<n$, then $M$ is isoparametric. Moreover, each leaf $M^{*}$ of $S(T M)$ is locally isometric to the product of spheres $\mathbb{S}^{m}\left(r_{1}\right) \times \mathbb{S}^{n-m}\left(r_{2}\right)$, where $\mathbb{S}^{m}\left(r_{2}\right)$ and $\mathbb{S}^{n-m}\left(r_{1}\right)$ are $m$ and $(n-m)$-spheres in $\bar{M}$, respectively. Furthermore, if $\bar{M}=\mathbb{R}_{1}^{n+2}$ then $M^{*}$ is locally isometric to a product of a spherical cylinder $\mathbb{S}^{m}(r) \times \mathbb{R}^{n-m}$.

Proof. Consider an orthonormal frame $X_{1}, \ldots, X_{m}, X_{m+1}, \ldots, X_{n}$, such that $1 \leq$ $i \leq m$ and $m+1 \leq j \leq n$,

$$
\begin{aligned}
& X_{i} \in P_{\lambda}=\left\{X \in S(T M)_{p}, \quad p \in M: A_{E}^{*} X=\lambda X\right\} \\
& \text { and } X_{j} \in P_{\mu}=\left\{X \in S(T M)_{p}, p \in M: A_{E}^{*} X=\mu X\right\} \text {. }
\end{aligned}
$$

In view of Proposition 3.7, we have $X_{i}(\lambda)=X_{j}(\mu)=0$. Then, by definition of mean curvature, we have

$$
n \mathcal{H}=\operatorname{tr}_{\mid S(T M)} B=m \lambda+(n-m) \mu .
$$

Differentiating (3.9) with respect to $E_{j}$ and taking into account that $\mathcal{H}$ is constant, we get $m E_{j}(\lambda)=-(n-m) E_{j}(\mu)=0$. Thus, $\lambda$ and $\mu$ are constant and $M$ is 
an isoparametric null hypersurface. In view of Theorem 3.9, the leaves $M_{\lambda}$ and $M_{\mu}$ with respect to the principal distributions $P_{\lambda}$ and $P_{\mu}$, respectively, are totally umbilic in $\bar{M}$ and totally geodesic in $S(T M)$. Since $S(T M)$ is Riemannian, we have $S(T M)=P_{\lambda} \perp P_{\mu}$, where the principal distributions $P_{\lambda}$ and $P_{\mu}$ are each parallel with respect to the connection on a leaf $M^{*}$ of $S(T M)$ (see $[1,14]$ for details). By the decomposition theorem of de Rham [9], we have $M^{*}=M_{\lambda} \times M_{\mu}$. As the leaves of $S(T M)$ are compact and each $M_{\lambda}$ and $M_{\mu}$ are metric spheres, we have that each leaf $M^{*}$ is isometric to $\mathbb{S}^{n-m}\left(r_{1}\right) \times \mathbb{S}^{m}\left(r_{2}\right) \subset \mathbb{R}^{n-m+1} \times \mathbb{R}^{m+1}$ (see [4] for more details). Next, when $\bar{M}=\mathbb{R}_{1}^{n+2}$, we know from [1] that one of the principal curtures is zero. Moreover, a simple calculation, using (2.9) and (2.10), reveals that the sectional curvatures of the underlying leaves $M_{0}$ and $M_{\lambda}$ from the two principal distributions $P_{0}$ and $P_{\lambda}$ are 0 and $2 \psi \lambda^{2}$, respectively. As $\psi$ is constant along each principal distribution (see Proposition 3.7), we conclude that both $M_{0}$ and $M_{\lambda}$ are of constant sectional curvatures 0 and $2 \psi \lambda^{2}$, respectively. Hence, $M^{*}$ splits as $\mathbb{S}^{m}(r) \times \mathbb{R}^{n-m}$, which completes the proof.

\section{GEODESICS AND ISOPARAMETRIC NULL HYPERSURFACES}

On a Riemannian manifold $\tilde{M}$, a smooth curve $\alpha: I \longrightarrow \tilde{M}$ is called a circle of curvature $\kappa$ if it is parametrized by its arc-length $s$ and it satisfies the following equation: $\tilde{\nabla}_{\alpha^{\prime}} \tilde{\nabla}_{\alpha^{\prime}} \alpha^{\prime}(s)=-\kappa^{2} \alpha^{\prime}(s)$, where $\kappa$ is constant and $\tilde{\nabla}_{\alpha^{\prime}}$ denotes the covariant differentiation along $\alpha^{\prime}$ with respect to the Riemannian connection $\tilde{\nabla}$ of $\tilde{M}$. As $\left\|\tilde{\nabla}_{\alpha^{\prime}} \alpha^{\prime}(s)\right\|=k$, this relation is equivalent to a geodesic when $\kappa=$ 0 . Consequently, a geodesic is looked at as circle of zero curvature (see [16] for more details). According to [16], if $\tilde{M}$ is embedded in $\bar{M}$ as an $n$-dimensional submanifold, then $\tilde{M}$ is called an extrinsic sphere if and only if every curve $\alpha$ on $\tilde{M}$ starting at a point $x \in \tilde{M}$ is developable upon a curve lying in a certain Euclidian $n$-sphere in $T_{p} \bar{M}$.

Let $M$ be a null hypersurface of $\bar{M}$. As above, the concept of circles and extrinsic spheres can in the same way be defined between a leaf of $S(T M)$ and $\bar{M}$. This follows easily since a leaf is a non-degenerate codimension 2 submanifold of $\bar{M}$. In general, this fails for a null hypersurface as a curve $\beta$ in $M$ may be null, spacelike or timelike, accordingly if $g\left(\beta^{\prime}, \beta^{\prime}\right)=0, g\left(\beta^{\prime}, \beta^{\prime}\right)>0$ or $g\left(\beta^{\prime}, \beta^{\prime}\right)<0$, respectively. In fact, let $\beta$ be a null curve in $M$, and set $E:=\beta^{\prime}$. Then, it is easy to show, using (2.3) and (2.6), that $\bar{\nabla}_{E} \bar{\nabla}_{E} E=\nabla_{E} \nabla_{E} E=-\left(E(\tau(E))+\tau(E)^{2}\right) E$, which renders the definition of circles, as seen for non-degenerate submanifolds, in $M$ for all curves $\beta$ null and void. Hence, let $(M, g)$ be a null hypersurface of $\bar{M}$, and $\beta$ be a spacelike or timelike curve in $M$, we say that $\beta$ is a degenerate circle 
in $M$ if

$$
\nabla_{\beta^{\prime}} \nabla_{\beta^{\prime}} \beta^{\prime}=-k^{2} \beta^{\prime},
$$

where $k$ is a constant along $\beta^{\prime}$ and will be called the curvature of $\beta$. Accordingly, $M$ is called a degenerate extrinsic sphere in $\bar{M}$.

As an example, we have the following.

Example 4.1. Consider the null cone $M$ of $\mathbb{R}_{1}^{3}$ given by

$$
M=\left\{\left(x^{1}, x^{2}, x^{3}\right) \in \mathbb{R}_{1}^{3}-\{0\}: x^{1}=\sqrt{\left(x^{3}\right)^{2}+\left(x^{2}\right)^{2}}\right\} .
$$

A routine computation reveals that $\operatorname{Rad} T M$ and $\operatorname{tr}(T M)$ are, respectively, spanned by

$$
\begin{aligned}
E & =x^{1} \partial x^{1}+x^{2} \partial x^{2}+x^{3} \partial x^{3}, \\
\text { and } N & =-\frac{1}{2\left(x^{1}\right)^{2}}\left(x^{1} \partial x^{1}-x^{2} \partial x^{2}-x^{3} \partial x^{3}\right) .
\end{aligned}
$$

It follows that the corresponding screen distribution $S(T M)$ is spanned by the unit spacelike vector field

$$
W=\frac{X^{2}}{\sqrt{\left(X^{2}\right)^{2}+\left(X^{3}\right)^{2}}} \partial x^{2}+\frac{X^{3}}{\sqrt{\left(X^{2}\right)^{2}+\left(X^{3}\right)^{2}}} \partial x^{3},
$$

where $X^{2}$ and $X^{3}$ satisfy $x^{2} X^{2}+x^{3} X^{3}=0$. By simple calculations, we get

$$
\nabla_{X} E=\bar{\nabla}_{X} E=X, \quad \forall X \in \Gamma(T M),
$$

due to the fact that $E$ is the position vector field on $M$. Consequently, $A_{E}^{*} X+$ $\tau(X) E+X=0$. Setting $X=W$ in this relation, we get $A_{E}^{*} W=-W$, i.e., $M$ is totally umbilic in $\mathbb{R}_{1}^{3}$. Furthermore, $\tau(X)=\bar{g}(X, N)$. It is easy to check that $\nabla_{E} X \in \Gamma(S(T M))$, thus $C(E, X)=0$, for all $X \in \Gamma(T M)$. On the other hand,

$$
\bar{\nabla}_{W} N=\frac{1}{2\left(x^{1}\right)^{2}} W, \quad \forall W \in \Gamma(S(T M)) .
$$

From (4.3) we deduce that $A_{N} W=-\frac{1}{2\left(x^{1}\right)^{2}} W$. From (4.2) and (4.3), we derive

$$
\bar{\nabla}_{W} W=-\frac{1}{2\left(x^{1}\right)^{2}} E-N, \quad \nabla_{W} W=-\frac{1}{2\left(x^{1}\right)^{2}} E, \quad \nabla_{W}^{*} W=0 .
$$

Applying $\nabla_{W}$ to (4.4) and then using (4.2), we get

$$
\nabla_{W} \nabla_{W} W=-\frac{1}{2\left(x^{1}\right)^{2}} W
$$


Hence, the spacelike curve $\beta$ for which $\beta^{\prime}=W$ is a degenerate circle in $M$. Consequently, $M$ is a degenerate extrinsic sphere in $\mathbb{R}_{1}^{3}$, with curvature $k=\frac{1}{\sqrt{2} x^{1}}$. Differentiating the first and last relations of (4.4), we get

$$
\bar{\nabla}_{W} \bar{\nabla}_{W} W=-\frac{1}{\left(x^{1}\right)^{2}} W \text { and } \nabla_{W}^{*} \nabla_{W}^{*} W=0,
$$

respectively. Thus, $\beta$ is a circle in $\mathbb{R}_{1}^{3}$, with curvature $\kappa=\frac{1}{x^{1}}$. On the other hand, from the second relation of (4.6), we can see that $\beta$ is a geodesic circle in $S(T M)$. Notice from the expressions of $k$ and $\kappa$ that $\beta$ looks like a geodesic circle in both $M$ and $\bar{M}$ at $\infty$.

We prove the following characterization result on isoparametric null hypersurfaces of $\bar{M}(c)$.

Theorem 4.2. Let $(M, g)$ be a screen conformal null hypersurface of $(\bar{M}(c=$ $0), \bar{g})$ and let $M^{*}$ be a leaf of its screen distribution $S(T M)$. Then $M$ is isoparametric in $\bar{M}(c)$ if and only if for each point $x \in M$ there exists an orthonormal basis $\left\{X_{i}\right\}_{i=1}^{m}$ of the orthogonal complement of $\operatorname{ker} A_{E}^{*}$ in $S(T M)_{x}$ such that all geodesics of $M^{*}$ through $x$ in the direction $X_{i}$ lie on circles of nonzero curvature in $\bar{M}(c)$. Moreover, in this case the null hypersurface $M$ is a degenerate extrinsic sphere in $\bar{M}(c)$.

Proof. Let $M$ be a screen conformal isoparametric null hypersurface of $\bar{M}(c=0)$. Theorem 3.9 suggests that there are at most two distinct principal curvatures $\lambda$ and $\mu$ with multiplicities $m$ and $(n-m)$ (say), respectively. In case $\lambda=\mu$, then $M$ is totally umbilic in $\bar{M}$ and the conclusion follows from Theorem 3.9. On the other hand, if $\lambda \neq \mu$, then one of them is zero (say $\mu$ ). Then, we recall (see Proposition 3.7) the fact that each distribution $P_{t}(x): t \in\{\lambda, 0\}$ is integrable and moreover, every leaf of $P_{t}(x)$ is totally umbilic in $\bar{M}(c)$ and totally geodesic in $S(T M)$ (see Theorem 3.9), which implies that every geodesic of such leaves is a circle in $\bar{M}(c)$. Furthermore, let $\beta_{i}=\beta_{i}(s): i \in\{1, \ldots, m\}$ be geodesics of $M^{*}$ such that $\beta_{i}(0)=x$ and $\beta_{i}^{\prime}(0)=X_{i}$, where $\{1, \ldots, m\}$ is an orthonormal basis of $\left(\operatorname{ker} A_{E}^{*}\right)^{\perp}$ in $S(T M)_{x}$. As $S(T M)$ is non-degenerate, we have $\nabla_{X_{i}}^{*} X_{i}=0$, and by a simple calculation, using the Gauss-Weingartein equations of $S(T M)$, we have $\nabla_{X_{i}} \nabla_{X_{i}} X_{i}=-\psi k_{i} A_{E}^{*} X_{i}=-\psi k_{i}^{2} X_{i}$. Clearly, this relation shows that each $\beta_{i}$ is a degenerate circle in $M$ with curvature $\sqrt{\psi} k_{i}$. Hence, for each point $x \in M$, taking an orthonormal basis $\left\{X_{i}\right\}_{i=1}^{m}$ of the orthogonal complement of $\operatorname{ker} A_{E}^{*}$ in $S(T M)_{x}$ in such a way that each $X_{i}: i \in\{1, \ldots, m\}$ is a principal curvature vector, we find that the vectors $\left\{X_{i}\right\}_{i=1}^{m}$ satisfy the statement of Theorem 4.2. 
Conversely, let $M$ be a null hypersurface satisfying the condition that for each point $x \in M$ there exists an orthonormal basis $\left\{X_{i}\right\}_{i=1}^{m}$ of the orthogonal complement of $\operatorname{ker} A_{E}^{*}$ in $S(T M)_{x}$ such that all geodesics of $M$ through $x$ in the direction $X_{i}: i \in\{1, \ldots, m\}$, lie on circles of nonzero curvature in the ambient space $\bar{M}(c)$. Consider the open dense subset $\mathcal{U}=\{x \in M$ : the multiplicity of each principal curvature of $A_{E}^{*}$ along each leaf $M^{*}$ in $\bar{M}(c)$ is constant on some neighborhood $\mathcal{V}_{x} \subseteq \mathcal{U}$ of $\left.x\right\}$ of $M$. Fix neighborhood $\mathcal{V}_{x}$. Notice that the shape operator $A_{E}^{*}$ has constant rank on $\mathcal{V}_{x}$. The curves $\beta_{i}$ satisfies

$$
\bar{\nabla}_{\beta_{i}^{\prime}} \bar{\nabla}_{\beta_{i}^{\prime}} \beta_{i}^{\prime}=-\kappa_{i}^{2} \beta_{i}^{\prime}, \quad \forall i \in\{1, \ldots, m\},
$$

for some positive constants $\kappa_{i}$. Let us assume, without loss of generality, that $\kappa_{1} \leq \kappa_{2} \leq \ldots \leq \kappa_{m}$. By simple calculations we have

$$
\bar{\nabla}_{\beta_{i}^{\prime}} \bar{\nabla}_{\beta_{i}^{\prime}} \beta_{i}^{\prime}=-2 \psi B\left(\beta_{i}^{\prime}, \beta_{i}^{\prime}\right) A_{E}^{*} \beta_{i}^{\prime}+\beta_{i}^{\prime}\left(\psi B\left(\beta_{i}^{\prime}, \beta_{i}^{\prime}\right)\right) E+\beta_{i}^{\prime}\left(B\left(\beta_{i}^{\prime}, \beta_{i}^{\prime}\right)\right) N .
$$

From (4.7) and (4.8), we get

$$
B\left(\beta_{i}^{\prime}, \beta_{i}^{\prime}\right) A_{E}^{*} \beta_{i}^{\prime}=\frac{\kappa_{i}^{2}}{2 \psi} \beta_{i}^{\prime}, \quad \beta_{i}^{\prime}\left(B\left(\beta_{i}^{\prime}, \beta_{i}^{\prime}\right)\right)=0, \quad \beta_{i}^{\prime}(\psi)=0,
$$

so that at $s=0$, the first relation of (4.9) reduces to $A_{E}^{*} X_{i}=\lambda_{i} X_{i}$ or $A_{E}^{*} X_{i}=$ $-\lambda_{i} X_{i}$, where $\lambda_{i}:=\frac{\kappa_{i}}{\sqrt{2 \psi}}$. Then, the last two relations of (4.9) reads as $X_{i}\left(\lambda_{i}\right)=0$ and $X_{i}(\psi)=0$, respectively. Consequently, at each $x \in M$, the tangent space $T_{x} M$ has the following decomposition $T_{x} M=T_{x} M^{\perp} \perp P_{0}(x) \perp P_{\lambda_{i_{1}}}(x)$ or $T_{x} M=T_{x} M^{\perp} \perp P_{0}(x) \perp P_{-\lambda_{i_{1}}}(x)$ where 0 and $\lambda_{i_{1}}$ are the two distinct $\lambda_{i}$ 's as per Theorem 3.9 and that $0<\lambda_{i_{1}}$. Hence, both 0 and $\lambda_{i_{1}}$ are differentiable on $\mathcal{V}_{x}$. Next, we shall show the constancy of $\lambda_{i_{1}}$, which suffices to check the case $A_{E}^{*} X_{i_{1}}=\lambda_{i_{1}} X_{i_{1}}$, for all $i \in\{1, \ldots, m\}$. By the method of [14] in Lemma 2.5.10, we have $\left.\left(d \lambda_{i_{1}}+\lambda_{i_{1}} \tau-\lambda_{i_{1}}^{2} \eta\right)\right|_{P_{\lambda_{i_{1}}}}(x)=0$. As $\tau=0$ on $S(T M)_{x}$, we get $d \lambda_{i_{1}}=0$ on $S(T M)_{x}$, which completes the proof.

As a consequence of Theorem 4.2, we have the following.

Corollary 4.3. Let $(M(\tilde{c}), g)$ be a screen conformal null hypersurface of $(\bar{M}(c), \bar{g})$ and let $M^{*}$ be a leaf of its screen distribution $S(T M)$. Then $M$ is isoparametric in $\bar{M}(c)$ with non-zero screen principal curvatures if and only if for each point $x \in M$ there exists an orthonormal basis $\left\{X_{i}\right\}: i \in\{1, \ldots, m\}$ of the orthogonal complement of $\operatorname{ker} A_{E}^{*}$ in $S(T M)_{x}$ such that all geodesics of $M^{*}$ through $x$ in the direction $X_{i}$ lie on circles of nonzero curvature in $\bar{M}(c)$. Moreover, the null hypersurface $M$ is a degenerate extrinsic sphere in $\bar{M}(c)$. 


\section{QUASI ISOPARAMETRIC NULL HYPERSURFACES}

We have seen that a null hypersurface is endowed with two shape operators $A_{E}^{*}$ and $A_{N}$. We have so far used the principal curvatures from $A_{E}^{*}$ to characterize isoparametric null hypersurfaces, since $A_{E}^{*}$ is a symmetric operator and therefore, diagonalizable. On the other hand, the operator $A_{N}$ is generally non-symmetric and therefore non-diagonalizable. However, if suitable geometric conditions are imposed on $M, A_{N}$ can be made symmetric on $M$. For instance, when $M$ is screen integrable null hyperserface, one can easily show that $A_{N}$ is symmetric (see details in Theorem 2.2.6 of [14, p. 55]). Other assumed geometric conditions are screen conformality, in which $A_{N}=\psi A_{E}^{*}$ for some non-vanishing smooth function $\psi$ on a neighborhood $\mathcal{U}(\subset M)$, and parallel screen distribution, which all make $A_{N}$ a symmetric operator on $M$. In this section, we will suppose that $A_{N}$ is symmetric. We will assume, further, that $A_{N} E=0$, i.e., $E$ is a principal vector field of $A_{N}$ with corresponding principal curvature 0 . This is motivated by the fact that the screen second fundamental form $C$ is degenerate and $\operatorname{rank} A_{N} \leq n$. In that line, we have the following definition.

Definition 5.1. A null hypersurface $(M, g)$ of a semi-Riemannian space form $(\bar{M}(c), \bar{g})$ is called quasi isoparametric if all the principal curvatures of $A_{N}$ are constant along $S(T M)$.

As examples; Any screen totally geodesic null hypersurface is quasi isoparametric with one screen principal curvature 0. A null cone of Example 3.3 is quasi isoparametric with one screen principal curvature $-1 / 2 x_{0}^{2}$.

Let $M$ be a screen integrable quasi isoparametric null hypersurface, with two distinct screen principal curvatures $\tilde{\lambda}$ and $\tilde{\mu}$ with multiplicities $m$ and $(n-m)$, respectively. Denote by $P_{\tilde{\lambda}}$ and $P_{\tilde{\mu}}$, the corresponding principle distributions. It is easy to show, from (2.12) and the fact that $\bar{M}$ is a space of constant curvature $c$, that both $P_{\tilde{\lambda}}$ and $P_{\tilde{\mu}}$ are integrable distributions. Moreover, each principle curvature $\tilde{\lambda}$, $\tilde{\mu}$ is constant along its corresponding principle distribution if and only if $\tau$ vanishes on $S(T M)$. Following the method of [14] in Lemma 2.5.6, one can show easily that $P_{\tilde{\lambda}} \perp_{g} P_{\tilde{\mu}}$. We will prove a classification result on quasi isoparametric null hypersurfaces of $\bar{M}(c=0)$. We will need the following two Lemmas.

Lemma 5.2. On a screen integrable null hypersurface $(M, g)$ of $(\bar{M}(c), \bar{g})$, with two distinct principal curvatures $\tilde{\lambda}$ and $\tilde{\mu}$ with respect to $A_{N}$, such that $\tau$ vanishes on $S(T M)$, we have

(1) $P_{\tilde{\lambda}} \perp_{B} P_{\tilde{\mu}}$; 
(2) For all $X \in \Gamma\left(P_{\tilde{\lambda}}\right), Y \in \Gamma\left(P_{\tilde{\mu}}\right)$ and $Z \in \Gamma(S(T M))$, we have

$$
g\left(\left(\nabla_{Z} A_{N}\right) X, Y\right)=(\tilde{\lambda}-\tilde{\mu}) g\left(\nabla_{Z} X, Y\right)
$$

(3) For all $X, Y \in \Gamma\left(P_{\tilde{\lambda}}\right)$, we have $\nabla_{X} Y \in \Gamma\left(P_{\tilde{\lambda}} \perp T M^{\perp}\right)$, on a quasi isoparametric null hypersurface $M$;

(4) $\nabla_{X} Y \perp P_{\tilde{\lambda}}$, if $X \in \Gamma\left(P_{\tilde{\lambda}}\right)$ and $Y \in \Gamma\left(P_{\tilde{\mu}}\right)$, on a quasi isoparametric null hypersurface $M$.

Proof. Since $\bar{M}$ is a space of constant curvature $c$, we have $\bar{R}(X, Y) N=0$, for any $X, Y \in \Gamma(S(T M))$. Consequently, (2.12) yields

$$
B\left(Y, A_{N} X\right)=B\left(X, A_{N} Y\right) \text { and }\left(\nabla_{X} A_{N}\right) Y=\left(\nabla_{Y} A_{N}\right) X
$$

for any $X, Y \in \Gamma(S(T M))$. Setting $X \in \Gamma\left(P_{\tilde{\lambda}}\right)$ and $Y \in \Gamma\left(P_{\tilde{\mu}}\right)$ in the first relation in (5.1), and using the symmetry of $B$, we obtain $(\tilde{\lambda}-\tilde{\mu}) B(X, Y)=0$. As $\tilde{\lambda} \neq \tilde{\mu}$, we see that $B(X, Y)=0$, i.e., $P_{\tilde{\lambda}} \perp_{B} P_{\tilde{\mu}}$, which proves (1). To prove (2), we first note that $\left(\nabla_{Z} A_{N}\right) X=\nabla_{Z} A_{N} X-A_{N} \nabla_{Z} X$, for any $X \in \Gamma\left(P_{\tilde{\lambda}}\right)$ and $Z \in \Gamma(S(T M))$. Thus, since $A_{N} E=0$, we have $g\left(\left(\nabla_{Z} A_{N}\right) X, Y\right)=$ $(\tilde{\lambda}-\tilde{\mu}) g\left(\nabla_{Z} X, Y\right)+Z(\tilde{\lambda}) g(X, Y)$, for any $Y \in \Gamma\left(P_{\tilde{\mu}}\right)$. As $P_{\tilde{\lambda}} \perp_{g}, P_{\tilde{\mu}}$, we obtain (2). Next, we prove (3). From the second relation of (5.1), we notice that $g\left(\left(\nabla_{X} A_{N}\right) Z, Y\right)=g\left(\left(\nabla_{Z} A_{N}\right) X, Y\right)$, for any $X, Y \in \Gamma\left(P_{\tilde{\lambda}}\right)$ and $Z \in \Gamma\left(P_{\tilde{\mu}}\right)$. Thus, by part (2), this relation reduces to $(\tilde{\mu}-\tilde{\lambda}) g\left(\nabla_{X} Z, Y\right)-Z(\tilde{\lambda}) g(X, Y)=0$. As $M$ is quasi isoparametric with $\tilde{\lambda} \neq \tilde{\mu}$, this last relation gives $g\left(\nabla_{X} Z, Y\right)=0$, which implies that $g\left(\nabla_{X}^{*} Y, Z\right)=0$, in which we have used (2.5). Hence, $\nabla_{X}^{*} Y \in$ $\Gamma\left(P_{\tilde{\lambda}}\right)$, and by considering (2.5), we obtain part (3). For part (4), we have already seen that $g\left(\nabla_{X} Z, Y\right)=0$, for any $X, Y \in \Gamma\left(P_{\tilde{\lambda}}\right)$ and $Z \in \Gamma\left(P_{\tilde{\mu}}\right)$, which completes the proof.

Next, with the aid of Lemma 5.2, we prove a Cartan-like identity on a quasi isoparametric null hypersurface, which we later use in our classification result.

Lemma 5.3. Let $(M, g)$ be a screen integrable quasi isoparametric null hypersurface of $(\bar{M}(c), \bar{g})$, such that $\tau$ vanishes on $S(T M)$. Let $X$ be a unit principal vector at a point $x \in M$ and $\tilde{\lambda}$ the associated principal curvature. For any orthonormal basis $\left\{X_{i}\right\}_{i=1}^{n}$ satisfying $A_{N} X_{i}=\tilde{\mu}_{i} X_{i}$, we have

$$
\sum_{\tilde{\mu}_{i} \neq \tilde{\lambda}} \frac{c+\tilde{\lambda} B\left(X_{i}, X_{i}\right)+\tilde{\mu}_{i} B(X, X)}{\tilde{\lambda}-\tilde{\mu}_{i}}=0 .
$$

Proof. Observe that when $A_{N} E=0$ then $\nabla A_{N}$ is symmetric on $S(T M)$. In fact, using (2.8) we see that $g\left(\nabla_{X} A_{N} Y, Z\right)=X g\left(A_{N} Y, Z\right)-g\left(A_{N} Y, \nabla_{X} Z\right)$, for any $X, Y, Z \in \Gamma(S(T M))$. By virtue of (2.5), we derive $g\left(\left(\nabla_{X} A_{N}\right) Y, Z\right)=$ 
$g\left(\nabla_{X} A_{N} Y, Z\right)-g\left(\nabla_{X} Y, A_{N} Z\right)$. Applying the two relations above, one gets $g\left(\left(\nabla_{X} A_{N}\right) Y, Z\right)=g\left(Y,\left(\nabla_{X} A_{N}\right) Z\right)$, for any $X, Y, Z \in \Gamma(S(T M))$. That is, $\nabla A_{N}$ is symmetric on $S(T M)$.

As per the lemma, let $Y$ be a second unit principal vector with corresponding principal curvature $\tilde{\mu} \neq \tilde{\lambda}$. Since $S(T M)$ is integrable and $\tau=0$ on $S(T M)$, we see, from (2.12), that

$$
\begin{aligned}
g\left(\left(\nabla_{[X, Y]} A_{N}\right) X, Y\right) & =g\left([X, Y],\left(\nabla_{X} A_{N}\right) Y\right) \\
& =(\tilde{\lambda}-\tilde{\mu}) g\left(\nabla_{X} Y, \nabla_{Y} X\right) .
\end{aligned}
$$

Since $\bar{M}$ is a space of constant curvature $c$ and that $\tau=0$ on $S(T M)$, relation (2.12) reduces to

$$
\begin{aligned}
R(X, Y) Z & =c\{g(Y, Z) X-g(X, Z) Y\}-B(X, Z) A_{N} Y \\
& +B(Y, Z) A_{N} X, \quad \forall X, Y, Z \in \Gamma(S(T M)) .
\end{aligned}
$$

Setting $X \in \Gamma\left(P_{\tilde{\lambda}}\right), Y \in \Gamma\left(P_{\tilde{\mu}}\right)$ and $Z=Y$ in (5.3) and using (1) of Lemma 5.2, we have

$$
g(R(X, Y) Y, X)=c+\tilde{\lambda} B(Y, Y) .
$$

Using (4) of Lemma 5.2 we see that $g\left(\nabla_{X} Y, X\right)=0$. In view of this relation and (2.8), we derive

$$
\begin{aligned}
g\left(\nabla_{Y} \nabla_{X} Y, X\right) & =-\left(\nabla_{X} g\right)\left(\nabla_{Y} Y, X\right)-g\left(\nabla_{Y} Y, \nabla_{X} X\right) \\
& =-B(X, X) \eta\left(\nabla_{Y} Y\right)-g\left(\nabla_{Y} Y, \nabla_{X} X\right) .
\end{aligned}
$$

By part (3) of Lemma 5.2, we have $g\left(\nabla_{Y} Y, \nabla_{X} X\right)=0$, and by direct calculations, using (2.5), we have $\eta\left(\nabla_{Y} Y\right)=C(Y, Y)=g\left(A_{N} Y, Y\right)=\tilde{\mu}$. Thus, (5.5) reduces to

$$
g\left(\nabla_{Y} \nabla_{X} Y, X\right)=\tilde{\mu} B(X, X) .
$$

Following a similar calculation, using part (4) of Lemma 5.2, (2.8), part (1) of Lemma 5.2, we derive

$$
g\left(\nabla_{Y} \nabla_{X} Y, X\right)=-g\left(\nabla_{X} Y, \nabla_{Y} X\right) .
$$

In view of the definition of the curvature tensor $R$, part (2) of Lemma 5.2, (5.6), (5.7) and (5.2), we see that

$$
g(R(X, Y) Y, X)=-\tilde{\mu} B(X, X)+2 g\left(\nabla_{X} Y, \nabla_{Y} X\right) .
$$

Then, from (5.5) and (5.8), we have

$$
2 g\left(\nabla_{X} Y, \nabla_{Y} X\right)=c+\tilde{\lambda} B(Y, Y)+\tilde{\mu} B(X, X) .
$$


On the other hand, let $Z$ be unit principal vector with corresponding principal curvature $\tilde{\nu}$ not equal to $\tilde{\lambda}$ or $\tilde{\mu}$, we have $g\left(\left(\nabla_{Z} A_{N}\right) X, Y\right)=(\tilde{\mu}-\tilde{\nu}) g\left(Z, \nabla_{X} Y\right)$ and inter-changing $X$ and $Y$ we get $g\left(\left(\nabla_{Z} A_{N}\right) X, Y\right)=(\tilde{\lambda}-\tilde{\nu}) g\left(Z, \nabla_{Y} X\right)$. From these two relations, we deduce that

$$
(\tilde{\lambda}-\tilde{\nu})(\tilde{\mu}-\tilde{\nu}) g\left(\nabla_{X} Y, Z\right) g\left(Z, \nabla_{Y} X\right)=g\left(\left(\nabla_{Z} A_{N}\right) X, Y\right)^{2} .
$$

Expressing the term $g\left(\nabla_{X} Y, \nabla_{Y} X\right)$ in the principal basis $\left\{X_{i}\right\}_{i=1}^{n}$, we see that

$$
g\left(\nabla_{X} Y, \nabla_{Y} X\right)=\sum_{\tilde{\mu}_{i} \neq \tilde{\lambda}, \tilde{\mu}} g\left(\nabla_{X} Y, X_{i}\right) g\left(X_{i}, \nabla_{Y} X\right) .
$$

Thus, from (5.9), (5.10) and (5.11), we have

$$
2 \sum_{\tilde{\mu}_{i} \neq \tilde{\lambda}, \tilde{\mu}} \frac{g\left(\left(\nabla_{X_{i}} A_{N}\right) X, Y\right)^{2}}{\left(\tilde{\lambda}-\tilde{\mu}_{i}\right)\left(\tilde{\mu}-\tilde{\mu}_{i}\right)}=c+\tilde{\lambda} B(Y, Y)+\tilde{\mu} B(X, X) .
$$

Observe that, for any $j$ with $\tilde{\mu}_{j} \neq \tilde{\lambda}$, we have by setting $Y=X_{j}$ in (5.12) and dividing by $\left(\tilde{\lambda}-\tilde{\mu}_{j}\right)$,

$$
\frac{c+\tilde{\lambda} B\left(X_{j}, X_{j}\right)+\tilde{\mu}_{j} B(X, X)}{\left(\tilde{\lambda}-\tilde{\mu}_{j}\right)}=2 \sum_{\tilde{\mu}_{i} \neq \tilde{\lambda}_{,} \tilde{\mu}_{j}} \frac{g\left(\left(\nabla_{X_{i}} A_{N}\right) X, Y\right)^{2}}{\left(\tilde{\lambda}-\tilde{\mu}_{i}\right)\left(\tilde{\mu}-\tilde{\mu}_{i}\right)\left(\tilde{\lambda}-\tilde{\mu}_{j}\right)} .
$$

Finally, by summing (5.13) over all $j$ for which $\tilde{\mu}_{j} \neq \tilde{\lambda}$ and observing that the resultant sum on the right is 0 , since it is skew-symmetric in $\{i, j\}$, we obtain the lemma.

Using the above lemmas, we prove the following result on quasi isoparametric null hypersurfaces.

Theorem 5.4. Let $(M, g)$ be a screen integrable quasi isoparametric null hypersurface of $\bar{M}(c=0)$, with two distinct screen principal curvatures. If $M$ is totally umbilical null hypersurface, then it is either an open subset of a hyperplane in $\bar{M}$ or its screen distribution is isometric to a Riemannian product $M_{\tilde{\lambda}} \times M_{-\tilde{\lambda}}$, where $M_{\tilde{\lambda}}$ and $M_{\tilde{\mu}}$ are leaves of $S(T M)$ of constant curvatures $2 \rho \tilde{\lambda}$ and $-2 \rho \tilde{\lambda}$, respectively. Moreover, if $M$ is screen conformal then $S(T M)$ is isometric to $M_{\tilde{\lambda}} \times \mathbb{E}$, where $M_{\tilde{\lambda}}$ is a space of constant curvature $2 \psi \tilde{\lambda}^{2}$ and $\mathbb{E}$ is an Euclidean space.

Proof. Let the two distinct principle curvatures be $\tilde{\lambda}$ and $\tilde{\mu}$, with multiplicities $m$ and $(n-m)$ respectively. Then, by Lemma 5.3, we have $c+\tilde{\lambda} B\left(X_{j}, X_{j}\right)+$ $\tilde{\mu} B\left(X_{i}, X_{i}\right)=0$, where $1 \leq i \leq m$ and $(m+1) \leq j \leq n$. If $M$ is umbilic and $c=0$, this relation gives $\rho(\tilde{\lambda}+\tilde{\mu})=0$. Thus, either $\rho=0$ in which case $M$ is totally geodesic in $\bar{M}$, hence showing that $M$ is an open subset of a hyperplane or $\tilde{\lambda}=-\tilde{\mu}$. In the second case, $S(T M)$ splits through two leaves $M_{\tilde{\lambda}}$ and $M_{-\tilde{\lambda}}$ with 
dimension $m$ each. It is easy to show, following the method of Theorem 3.9, that the two leaves are each totally umbilic in $\bar{M}$ and totally geodesic in $S(T M)$ (see part (3) of Lemma 5.2). Consequently, they are $m$-spheres in $\bar{M}$ and $m$-planes in $S(T M)$. By a simple calculation, using (2.9) and (2.10), we see that the sectional curvatures of $M_{\tilde{\lambda}}$ and $M_{-\tilde{\lambda}}$ are $2 \rho \tilde{\lambda}$ and $-2 \rho \tilde{\lambda}$, respectively. Observe that both sectional curvatures are constant along each leaf, since $M$ is totally umbilic and $\tau=0$ on $S(T M)$. In fact, as $M$ is umbilic, we have $P X(\rho)+\rho \tau(P X)=0$ (see [13]). That is, $\rho$ is a constant on $S(T M)$. On the other hand, if $M$ is screen conformal then $B=\frac{1}{\psi} C$. From Lemma 5.3 we have $c+\frac{2}{\psi} \tilde{\lambda} \tilde{\mu}=0$. As $c=0$, we see that one of the two principal curvatures is 0 . Let $\tilde{\lambda}$ be the non-zero principal curvature. As before, the sectional curvature of $M_{\tilde{\lambda}}$ is $2 \psi \tilde{\lambda}^{2}$ and that of $M_{0}$ is 0 , which completes the proof.

\section{REFERENCES}

[1] Atindogbé, C., Harouna, M. M., Tossa, J.: Lightlike hypersurfaces in Lorentzian manifolds with constant screen principal curvatures. Afr. Diaspora J. Math 16(2), 31-45 (2014).

[2] C. Atindogbé, Scalar curvature on lightlike hypersurfaces, Balkan Society of Geometers, Geometry Balkan Press 2009, Applied Sciences, Vol.11, 2009, pp. 9-18.

[3] Atindogbé, C., Ezin, J.-P. and Tossa, J. Pseudo-inversion of degenerate metrics Int. J. Math. Math. Sci., 55 (2003), 3479-3501.

[4] L. J. Alíias, S. C. De Almeida and A. Brasil Jr., Hypersurfaces with constant mean curvature and two principal curvatures in $\mathbb{S}^{n+1}$, Anais da Academia Brasileira de Cieéncias (2004) 76(3): 489-497.

[5] J. Berndt, Real hypersurfaces with constant principal curvatures in complex hyperbolic space, J. Reine Angew. Math., 395 (1989), 132-141.

[6] E. Cartan, Familles de surfaces isoparametriques dans les espaces a courbure constante, Ann. Mat. Pura IV 17, 177-191, (1938).

[7] B. Y. Chen, Riemannian submanifolds: A survey, https://arxiv.org/abs/1307.1875.

[8] E. Cartan, Sur quelques familles remarquables d'hypersurfaces, C. R. Congrés Math. Liége 30-41, (1939); Oeuvres completes Tome III, Vol. 2, 1481-1492.

[9] G. de Rham, Sur la réductibilité d'un espace de Riemann Comment. Math. Helv. 268, 328-344, (1952).

[10] J. Dong and X. Liu, Totally Umbilical Lightlike Hypersurfaces in Robertson-Walker Spacetimes, Hindawi Publishing Corporation, Volume 2014, Article ID 974695, 10 pages.

[11] M. Hassirou, Kaehler lightlike submanifolds, Journal of Mathematical Sciences: Advances and Applications, Volume 10, Number 1/2, 2011, Pages 1-21.

[12] J. Hahn, Isoparametric hypersurfaces in the pseudo-Riemannian space forms. Math. Z. 187, 195-208 (1984).

[13] K. L. Duggal and A. Bejancu, Lightlike submanifolds of semi-Riemannian manifolds and applications, Mathematics and Its Applications, Kluwer Academic Publishers, 1996.

[14] K. L. Duggal and B. Sahin, Differential geometry of lightlike submanifolds. Frontiers in Mathematics, Birkhäuser Verlag, Basel, 2010. 
[15] D. H. Jin, Ascreen lightlike hypersurfaces of an indefinite Sasakian manifold. J. Korean Soc. Math. Educ. Ser. B: Pure Appl. Math. Volume 20, No. 1 (2013), 25-35.

[16] M. Kimura and S. Maeda, Geometric meaning of isoparametric hypersurfaces in a real space form, Canad. Math. Bull. Vol. 43 (1), (2000), 74-78.

[17] Z.-Q. Li, X.-H. Xie, Spacelike isoparametric hypersurfaces in Lorentzian space forms. Translated from the Chinese original [J. Nanchang Univ. Natur. Sci. Ed. 28, 113-117 (2004)]. Front. Math. China 1. 130-137 (2006).

[18] M. Megid, Lorentzian isoparametric hypersurfaces, Pacific J. Math., 118(1) (1985), 165-197.

[19] F. Massamba and S. Ssekajja, Quasi generalized CR-lightlike submanifolds of indefinite nearly Sasakian manifolds, Arab. J. Math. 5 (2016) 87-101.

[20] B. ONeill, Semi-Riemannian Geometry, with Applications to Relativity. New York: Academic Press (1983).

[21] K. Nomizu, Élie Cartan's work on isoparametric families of hypersurfaces, in Proceedings of Symposia in Pure Mathematics, vol. 27, Part 1 (American Mathematical Society, Providence, 1975), 191-200.

[22] K. Nomizu, Some results in E. Cartans theory of isoparametric families of hypersurfaces, Bull. Amer. Math. Soc., 79 (1973), 1184-1188.

[23] M. Navarro, O. Palmas and D. A. Solis, Null screen isoparametric hypersurfaces in Lorentzian space forms, Mediterr. J. Math. (2018) 15:215.

[24] D. N. Kupeli, Singular semi-Riemannian geometry, Mathematics and Its Applications, Vol. 366, Kluwer Academic Publishers, 1996.

[25] Thomas E. Cecil and Patrick J. Ryan, Geometry of hypersurfaces, Springer Monographs in Mathematics. Springer, New York, 2015.

1 School of Mathematics, Statistics and Computer Science

UNIVERSITY OF KWAZULU-NATAL

Private BAG X01, SCOTTSVILle 3209

SOUTH AFRICA

Email address: ssekajja. samuel. buwaga@aims-senegal.org 